\title{
A Method of Dividing the Circumference of a Circle into 360 equal parts.
}

\author{
By James N. Miller.
}

This Graduation of the Circumference of a Circle is effected by the aid of an instrument called a trisector which $I$ contrived with the view of trisecting an angle by its assistance, but subsequently perceived that it could help to divide an angle into 5 equal angles, and recently discovered that it could contribute towards dividing the circumference of a circle into 360 equal degrees or arcs.

That instrument is outlined in the diagram (Fig. 32). It consists, as was formerly explained to this Society, of two flat or nearly flat pieces BEFGD and AHJ of peculiar forms; and may be made of metal or other material. Those two pieces are conjoined, as the blades of a pair of scissors are, by a small cylindrical pin inserted in a small cylindrical hole at $\mathrm{C}$ in each of them, which it fits, and round which they can be moved in either direction. A similar hole is made through the piece $B E F G D$ at $B$. The centres of those holes at $B$ and $C$, and also the points $E$ and $D$ are all in the same straight line BECD. An arm EFG projects from one of the sides of this piece. The edge EF of that arm is straight, and is perpendicular to the line $B C$ which joins the centres of the holes at $B$ and $C$. It also bisects the line $\mathrm{BC}$ in the point $\mathrm{E}$. The line $\mathrm{BE}$ is therefore equal to the line EC.

The point $\mathrm{A}$ of the other piece $\mathrm{AHJ}$, the centre of the hole at $\mathrm{C}$, and its edge IJ, are all in the straight line ACIJ. The line AC, drawn from the point $A$ to the centre at $C$, is exactly equal to the line $B C$ which connects the centres at $B$ and $C$. The triangle ACB is therefore isosceles.

The circumference of the circle, which it is proposed to graduate, may in the first instance be divided, as is easily done, into 3 equal arcs of $120^{\circ}$. Let MS be such an arc of $120^{\circ}$, B the centre of its circle, and $B M$ and $B S$ radii of the circle drawn to the extremities $M$ and $S$ of the arc. Also, let $K L$ be a perpendicular to the radius $B S$ drawn from the point $K$ in it, $K$ being at the same distance 
from the centre at $B$ as the point $E$ is from the centre of the hole at $B$. The lines $\mathbf{E B}$ and $\mathbf{K B}$ are therefore equal.

In employing the instrument, with a view to the graduation of the circumference of the circle, a sharp cylindrical pin which fits, but not tightly, the hole at $B$, is inserted through that hole into the centre $B$ of the circle, and at right angles to its plane. The point $A$ of the piece $A H J$ is then moved along the radius $B M$ until its edge IJ meets the edge EF of the arm EFG at a point, which may be termed $O$, on the perpendicular KL. Next, through $O$ draw the radius $B O Q$, and bisect the angle $C B O$ or $N B Q$ with the radius $B P$ and the angle $O B S$ or $Q B S$ with the radius $B R$; and produce the line BECD till it meets the arc MS at $N$.

Now, as by construction, the sides $\mathrm{BE}$ and $\mathrm{CE}$ of the rightangled triangles $\mathrm{BOE}$ and $\mathrm{COE}$ are equal, and as $\mathrm{EO}$ is a corresponding side of both those triangles, so their corresponding angles $\mathrm{CBO}$ or $\mathrm{NBQ}$ and $\mathrm{BCO}$ are equal. But, as $\mathrm{ACB}$ is an isosceles triangle, so its angle $A B C$ or $M B N$ is equal to half of its exterior angle $\mathrm{BCO}$. The angle $\mathrm{ABC}$ or $\mathrm{MBN}$ is therefore equal to each of the angles NBP and PBQ, they being halves of the angle NBQ, which has just been shown to be equal to the angle BCO. Thus the 3 angles MBN, NBP and PBQ are all equal.

Again, the sides $\mathrm{BK}$ and $\mathrm{BE}$ of the right-angled triangles $\mathrm{BOK}$ and $\mathrm{BOE}$ are, by construction, equal to each other ; and, as these triangles have the same hypotenuse $\mathrm{BO}$, so their corresponding angles $\mathrm{OBK}$ and $\mathrm{OBE}$ or $\mathrm{NBQ}$ are also equal to each other; and therefore their halves RBS, QBR, $P B Q$ and NBP are equal to qne another and to the angle MBN. Consequently the 5 arcs $R S, Q R, P Q, N P$ and MN by which those 5 equal angles are subtended, are all equal to each other. Each of those arcs is therefore a fifth of the arc MS of $120^{\circ}$, and is accordingly an arc of $24^{\circ}$.

Further, each of those 5 arcs may be divided into 3 equal arcs of $8^{\circ}$. To do this the trisector is moved round the pin at $B$, and the point $A$ of the piece $A H J$ is moved along the radius $B R$ until, when it is at a point $A^{\prime}$, the edges $I J$ and $E F$, which may now be denoted by $I^{\prime} J^{\prime}$ and $E^{\prime} F^{\prime}$, meet at a point which may be termed $\mathrm{O}^{\prime}$ on the radius BS. Now, through the centre at $\mathrm{C}^{\prime}$ in the piece $A^{\prime} H^{\prime} J^{\prime}$ draw the radius $B C^{\prime} N^{\prime}$, and bisect the angle $\mathrm{N}^{\prime} \mathrm{BS}$ with the radius $B T$. The arc $R S$ is thereby divided into 3 equal arcs $\mathrm{RN}^{\prime}, \mathrm{N}^{\prime} \mathrm{T}$, and TS. For, as it was proved that the angle $\mathrm{ABC}$ or 
MBN is equal to each of the angles $N B P$ and $P B Q$, so it may similarly be shown that the angle $A^{\prime} B C^{\prime}$ or $\mathrm{RBN}^{\prime}$ is equal to each of the angles $\mathrm{N}^{\prime} \mathrm{BT}$ and TBS. Consequently the 3 arcs $\mathrm{RN}^{\prime}, \mathrm{N}^{\prime} \mathrm{T}$ and TS, of which the arc RS of $24^{\circ}$ consists, and which subtend those equal angles, are equal to each other. Therefore each of them is an $\operatorname{arc}$ of $8^{\circ}$.

The other 4 arcs MN, NP, PQ and QR of $24^{\circ}$ each, may each be similarly divided into 3 arcs of $8^{\circ}$, and thereby the entire arc MS of $120^{\circ}$ would be divided into 15 arcs of $8^{\circ}$. Each of those 15 arcs may then be easily divided into 2 arcs of $4^{\circ}$, which may be divided into 4 arcs of $2^{\circ}$, which may be further divided into 8 arcs of $1^{\circ}$. The entire arc MS would thereby be divided into 120 equal arcs of $1^{\circ}$.

The other 2 arcs of $120^{\circ}$ may each be similarly divided into 120 arcs of $1^{\circ}$. In other words, the entire circumference of the cirele may be thus divided or graduated into 360 equal arcs of $1^{\circ}$. 